\title{
DE L'ESPAGNE AU QUÉBEC (1937 ET 1963): CONTINUITÉ CULTURELLE
}

\author{
CLAUDE PILLET
}

Université Sorbonne Paris IV

\section{Résumé}

Si Malraux quitte l'Espagne au début 1937, c'est pour défendre la cause républicaine par le verbe en Amérique du Nord. Cependant, comme à Montréal le 4 avril, ce ne sont pas les enjeux politiques et idéologiques de la guerre qu'il présente, mais le caractère universel, quasi métaphysique, de la lutte contre ce qui ignore ou méprise l'humanité. Curieusement quand Malraux revient au Canada en 1963, le contexte politique et diplomatique est suspendu de la même façon pour ne laisser entrevoir que ce domaine plus essentiel où se joue le destin de l'humanité que le ministre nomme «Culture».

Mots clés : République espagnole. Guerre civile espagnole. Canada. Québec. Discours politiques. Politique. Histoire. Culture.

\begin{abstract}
Early 1937, Malraux leaves Spain in order to plead the Republican cause in North America. Yet, as was the case in Montreal on April 4th, he underlines neither the political nor the ideological stakes of war, but the universal and almost metaphysical aspect of the struggle against whatever ignores or despises humanity. Interestingly enough, when Malraux returns to Canada in 1963, the political and diplomatic context is similarly put aside, only to highlight a more essential field that challenges the destiny of humanity, and which the minister calls "Culture".
\end{abstract}

Key words: Spanish Republic. Spanish Civil War. Canada. Quebec. Political speeches. Politics. History. Culture.

Après la mission de Teruel du 27 décembre 1936 (un mort, quatre blessés graves, un avion abattu) et celle du 11 février 1937 (chute d'un avion dans la mer), l'Escadrille André Malraux, considérablement diminuée, est dissoute (en huit mois d'activité, elle n'a jamais été que fort réduite). Ce n'est pas pour autant que va cesser l'engagement de l'écrivain auprès des Républicains. Alors même qu'il termine L'Espoir (le livre sortira de presse en décembre 1937), il renoue avec les grands meetings politiques. C'est d'abord Paris, sous l'égide de l'Association internationale des écrivains pour la défense de la culture qu'il connaît bien (Palais de la Mutualité, le $1^{\text {er }}$ février), puis ce très étonnant voyage en Amérique du Nord qui suscite alors une abondance de

\footnotetext{
${ }^{1}$ Université Sorbonne Paris IV. Correo: pillet@bluewin.ch. Recibido: 12-12-2011. Aceptado: 29-04-2012.
} 
commentaires. Durant six semaines, de la mi-février au début d'avril, Malraux parcourt le continent: New York (24 février - 3 mars), Philadelphie (5 mars), Washington (6 mars), Cambridge, Harvard (8 mars), New York à nouveau (11 mars) Princeton (12 mars), New York encore (13-20 mars), Los Angeles, Hollywood (22 mars), San Francisco (27 mars), Toronto (2 avril), Montréal (3-4 avril). Durant son séjour, Malraux proposera une vingtaine d'allocutions, conférences de presse, discours ou interviews.

On le voit, la tournée que Malraux s'impose est vaste et ambitieuse. Partout où il se rend, il suscite enthousiasme et réprobation, simultanément. Ce qui est curieux, c'est que l'une et l'autre ont été exprimées avec la même force et la même détermination. Invité par des associations de gauche, Malraux est ovationné par ses auditeurs, acquis d'avance à la cause républicaine; suspecté d'être un agent de Staline, l'écrivain est vilipendé par la presse proche des milieux politiques majoritaires et conservateurs. Plus encore parfois: il est considéré à ce point comme un «dangereux Rouge» que l'ambassade des États-Unis lui a refusé son visa dans un premier temps. Car l'affaire paraît sérieuse à tous: Malraux qui a combattu en Espagne (et en Chine comme on le croyait parfois), continue la lutte par le verbe et porte haut les couleurs républicaines et la cause qu'elles symbolisent. Il utilise tous les moyens que sa longue pratique de l'art oratoire met à sa disposition. La charge émotionnelle de ses images et hypotyposes est puissante et agissante; sa voix, ses mouvements, son intonation, le débit de ses phrases, la succession ou l'alternance d'évocations de faits vécus et de commentaires qui ne prennent à témoin que la justice universelle et la fraternité humaine la plus noble, tout cela que la rhétorique ancienne appelait «mœurs» touche immanquablement les «passions» de l'auditoire. Car nous avons affaire ici à l'efficace d'une propagande des plus habiles et des plus entraînées. Tout se passe en effet comme si le Malraux de 1937, aux États-Unis et au Canada, comme précédemment à Paris et comme à son retour en Europe en avril, comme si Malraux agissait en tant qu'émissaire ou comme le ministre de l'Information de la République espagnole.

Le cas de Montréal nous intéresse particulièrement parce qu'il est le moins connu, Robert S. Thornberry et Walter G. Langlois ayant éclairé de leurs irremplaçables recherches les séjours que Malraux a vécus en Amérique anglophone. ${ }^{2}$ On sait que Malraux, quel que soit son public, s'exprime en français et qu'un interprète traduit ses textes, parfois phrase après phrase ou paragraphe après paragraphe. On sait aussi que toujours Malraux improvise (à partir de quelques notes ou non), ce qui donne un aspect très «naturel» à son discours et renforce encore le pouvoir de son charisme (nous avons parlé plus haut des mœurs de l'orateur). Puisque dans le cas de Montréal Malraux n'est pas interrompu (la traduction anglaise n'aura lieu qu'une fois le discours terminé), nous avons peut-être affaire ici à l'un des textes les plus puissants que l'écrivain ait prononcé en Amérique.

\footnotetext{
${ }^{2}$ Signalons une étude historique excellemment documentée qui vient de paraître dans la revue électronique Présence d'André Malraux sur la Toile. Caroline Désy, «Malraux à Montréal : tensions et démesure autour d'une visite», Présence d'André Malraux sur la Toile, art. 115, décembre 2011. URL: $<$ http://www.malraux.org/index.php/articles/1507-art115.html>.
} 
Le texte n'ayant pas été écrit par Malraux ni enregistré comme celui de Columbia (20 mars 1937), notre source n'est malheureusement constituée que de fragments que publia Le Canada le 5 avril, extraits fâcheusement pourvus de titres intermédiaires et parfois de résumés. ${ }^{3}$

La veille, Malraux est intervenu à l'Eglise presbytérienne américaine de Montréal devant 1200 personnes. Le Comité d'aide médical à l'Espagne a organisé l'événement. La présence du $\mathrm{D}^{\mathrm{r}}$ Béthune à la tête de cette organisation empêcha sans doute la municipalité de Montréal d'appliquer la Loi du cadenas qui permet au procureur général de faire fermer tout bâtiment lié à la propagande communiste, article du code criminel qui avait permis de multiplier les chicanes à Toronto, où l'on dut changer de salle. Le $\mathrm{D}^{\mathrm{r}}$ Norman Béthune bénéficiait d'une grande popularité au Québec: il avait créé en Espagne «El instituto hispano-canadiense de transfusión de sangre» qui suscitait beaucoup d'admiration.

Malraux intervient donc à Montréal le 4 avril 1937, durant 30 minutes environ. Il y parle évidemment de la guerre; mais sa guerre n'est pas celle des combats sur le terrain ou dans les airs: elle est celle que subissent les enfants qui refusent les avionsjouets qu'on leur propose au moment où vient de se terminer un bombardement franquiste. Si elle est celle des bombardements de l'Escadrille André Malraux, ils ne sont que ceux qui détruisent des cibles militaires tandis que les bombardements fascistes dévastent les villes et en massacrent les habitants.

Quand il s'agit de l'Eglise, Malraux n'accuse personne pour constater que l'Eglise basque «s'est placée au-dessus de la toute politique» comme Pie XI lui-même et que si l'Eglise catalane agit tout inversement, c'est qu'elle a oublié depuis longtemps «d'enseigner l'amour». Si Mussolini prépare un affrontement avec l'Angleterre, c'est Franco, allié idéologique, qui le facilite. Si le fascisme divise et sépare, exclut et méprise (c'est cela, le «temps du mépris»), les Républicains s'ouvrent sur l'universel et rendent possible la «conquête de la culture» qui n'est plus un héritage réservé à une classe ou à un groupe privilégiés.

Et s'il s'agit du but de la guerre ou de sa raison d'être (même si la guerre ellemême est une détestation; Malraux écrira peu après: «Ah! que la victoire demeure avec ceux qui auront fait la guerre sans l'aimer! $\left.\gg^{4}\right), c^{\prime}$ est le combat qui transcende l'affrontement avec l'ennemi réel pour devenir le combat contre l'ordre du monde éternel qui oublie l'humanité, c'est la fraternité qui unit les hommes à leur vaste passé et au monde qui les ignore. C'est la descente de Valdelinares après le crash du Potez-N piloté par Florein (au cours duquel Raymond Maréchal est gravement blessé au visage), qui deviendra la grandiose scène de la «Descente de la montagne» de L'Espoir:

Je relevais la tête, dit Malraux à Montréal : la file des paysans s'étageait maintenant du haut en bas de la montagne. Et c'est la plus grande image de la fraternité que j'aie rencontrée de ma vie, ces villages abandonnés, ce peuple entier qui suivait des hommes blessés pour lui, et qu'il

\footnotetext{
${ }^{3}$ Voir l'annexe.

${ }^{4}$ La Lutte avec l'Ange, Lausanne, Le Haut-Pays, 1943, p. 223 ou CEuvres complètes, t. II, éd. de M.-F. Guyard, Paris, Gallimard 1996, p. 756.
} 
n'avait jamais vus, descendant comme une procession antique, avec ces sanglots qui montaient dans le grand silence des gorges - avec le bruit d'une rivière souterraine. (Malraux, 196: 756)

Impossible, semble-t-il, de ne pas comprendre où se trouvent l'homme et la légitimité des combats. Ce qui n'est pas curieux, c'est que les «adversaires de l'humanité» ont condamné Malraux avant son discours. Impossible pour eux de dissocier «communiste» (au sens où l'entendent ses adversaires anglo-saxons les plus acharnés) et «mal» (au sens où le comprennent les plus dogmatiques en matière de religion), impossible pour eux de comprendre que ce communisme est l'incarnation fictive (un moyen littéraire) qui s'associe à la libération de la dignité humaine. Louis Francœur écrit dans La Patrie du 4 avril:

[...] dire à Malraux et à ses pareils de nous ficher la paix, mettre l'opinion en garde contre eux, les montrer pour ce qu'il sont (fauteurs de désordre, amis d'assassins, etc.), leur refuser la permission de prêcher ici la destruction, c'est faire œuvre nécessaire, salubre et bienfaisance sans tenir compte des gloussements qui sortent de certains salons ou désaxées pernicieuses et «libérés» poseurs jouant avec la dynamite.

Sans doute de tels propos voulaient contredire ceux du Canada du 2 avril où l'on lit:

[...] les honnêtes gens qui ne sauraient entendre les directives de l'écrivain français ne peuvent pourtant s'empêcher de l'estimer, voire de l'admirer, puisqu'il est également un modèle de sincérité ou de probité intellectuelle. (Canada, 1937 : 16)

Il y a chez Malraux ambassadeur de la cause républicaine quelque chose d'aussi irrépressible que ce que met en scène $L$ 'Espoir: non un combat politique opposant deux camps («le nôtre et le leur»), pas davantage non plus une lutte idéologique comme le croient presque tous ses commentateurs québécois, mais la volonté de hisser l'enjeu de l'engagement là où les antagonismes de partis n'ont plus cours, là précisément où l'on ne considère que l'homme, son destin et sa volonté.

Quand Malraux visite le Québec en octobre 1963 et qu'il y prononce maints discours en tant que ministre des Affaires culturelles, ses propos ont dû étonner plus d'un auditeur. S'il y est venu pour inaugurer une exposition d'artistes français au musée des Beaux-Arts de Québec et l’Exposition française au Palais du Commerce de Montréal, il est reçu par la province comme le représentant personnel de De Gaulle. Aux journalistes qui le harcèlent à l'aéroport (Malraux y arrive de Paris le 7 et doit se rendre à Ottawa), il parle de la communauté française «qui ne connaît pas de frontières»; à l'Hôtel de Ville de Montréal (le 10), il déclare que «dans le destin du monde», «il y aura le poids de l'esprit français» duquel ses interlocuteurs font partie; à l'île Sainte-Hélène (le 14), il affirme qu' «Aujourd'hui commence dans l'Histoire du monde la volonté d'un certain nombre de pays de la vieille Europe [...] d'être ce qu'ils sont et de donner forme à leur âme et à leur rêve.» "C'est là dessus que nous nous unissons.», ajoutet-il. Car depuis la fin de la guerre d'Espagne et la Seconde Guerre Mondiale, l'enjeu des grands problèmes du monde - qui paraissent nécessairement comme politiques, économiques, démographiques, idéologiques ou stratégiques - appartient plus que jamais à un registre tout autre. Ce n'est pas celui où nations et alliances militaires s'affrontent, ni celui où les gouvernements et les diplomates rivalisent de ruses ou 
de calculs, ni non plus celui où des accords politiques se nouent ou se voient aussitôt trahis, mais bien celui qu'avait mis en évidence l'engagement espagnol de Malraux. L'Espoir même, d'un bout à l'autre, en était constamment saisi jusqu'à la scène de la «Descente de la montagne» où l'ennemi n'est bien évidemment plus franquiste ni militaire. Seule la mort reste contre laquelle s'élève la fraternité de Valdelinares.

Au moment où Malraux abandonne la pratique du roman, il se sépare simultanément de cette nécessité d'incarner dans une cause concrète le combat universel. S'il rallie le général de Gaulle, c'est justement que l'enjeu romanesque précédent passe maintenant sur la scène de l'Histoire, ce domaine quasi fictif des Grandes Figures et des Héros - où s'est joué d'ailleurs bien souvent le destin de la France. L'Histoire, en effet, pourtant aussi puissamment vraie que la réalité, est ce Grand Récit de l'humanité conçu presque comme de la fiction ou tout au moins établi en lui empruntant ses principales modalités. Là est sans doute l'un des traits les plus audacieux de la pensée de Malraux: considérer que la culture (il nomme ainsi le nouvel enjeu spirituel et intellectuel du monde) est précisément ce monde de formes et d'idées qui supplante l'autre - celui où l'on naît et meurt - parce sa vocation est de dépasser incessamment les conditionnements biologiques et les misères psychologiques de l'individu.

\section{ANNEXE}

Deux discours de Malraux (publiés avec l'aimable autorisation de Madame Florence Malraux).

\section{DISCOURS DE MONTRÉAL, 4 AVRIL 1937 (VERSION INÉDITE)}

Allocution prononcée à l'American Presbyterian Church, Montréal devant plus de 1200 personnes.

Principaux extraits du discours de Malraux, tirés du compte rendu paru dans Le Canada de Montréal, 5 avril 1937, p. 3 et 16.

\subsection{Le bombardement du Jour de l'An}

«A Madrid, au Jour de l'An, on distribua les jouets qui, de tous les pays du monde, avaient été envoyés aux enfants espagnols. La distribution avait lieu au centre des arènes, les jouets amoncelés par petits tas, enchevêtrés comme des insectes.

«Depuis une heure, les enfants passaient en silence devant ces petits tas où semblait s'être accumulée aussi l'affectueuse générosité de tous les coins du monde, quand commença l'éclatement des premières bombes. Une escadrille de Junkers bombardait. 
«Les bombes tombaient à six cents mètres. Le bombardement fut très court et les arènes sont très grandes. Quand les enfants qu'on évacuait arrivèrent aux portes des arènes, les avions étaient repartis et les enfants revinrent chercher les derniers jouets.

«Quand la distribution fut terminée, au centre des immenses arènes vides, il restait seulement un tas. Intact. Je m'approchai pour l'examiner : c'était le tas des avionsjouets. Il restait là, seul dans les arènes maintenant abandonnées et où n'importe quel gosse aurait pu venir. Les petits garçons avaient préféré n'importe quoi, même les poupées, et s'en étaient écartés, non avec peur, mais avec une sorte de mystérieuse horreur. Ce spectacle m'est resté dans la mémoire. Les fascistes et nous, nous sommes séparés à jamais par ce petit tas de jouets abandonnés.

«La terreur des bombardements aériens par les insurgés ébranle effroyablement les nerfs des enfants, et c'est ainsi toute une génération qu'on sacrifie.

«Je sais du reste que toute guerre est violente. Je sais du reste qu'il peut arriver que la bombe d'un avion gouvernemental qui vise un objectif militaire, tombe à côté et blesse des civils. Ce sur quoi je veux attirer votre attention de la façon la plus formelle, et vous le lirez dans les journaux fascistes eux-mêmes, c'est que nous avons détruit l'aérodrome de Séville, nous n'avons pas bombardé Séville. Nous avons détruit l'aérodrome de Salamanque, nous n'avons pas bombardé Salamanque. J'ai détruit l'aérodrome d'Avila, je n'ai pas bombardé Avila. Mais voilà plusieurs mois que les fascistes bombardent chaque jour les rue de Madrid.»

\subsection{Fascisme et démocratie}

«Quel est, dit-il, l'élément positif des fascistes ? C'est l'exaltation des différences essentielles, irréductibles et constantes : la race, la classe, la nation. Mais nous, nous voulons un Etat qui porte à un nombre de plus en plus grand d'hommes les connaissances, la culture et la dignité. Nous ne croyons pas que la culture soit un privilège. Elle appartient à tous les hommes. Nous attendons plus pour la culture de ceux qui l'appellent que de ceux qui l'ont simplement héritée.

«J'ai entendu, continue-t-il, le poète Alberti réciter ses poèmes aux arènes devant 200,000 paysans silencieux. J'ai vu la foule de Paris commémorer la mort de Gorki et je pensais à la même foule qui dans tous les pays du monde entourait à la même heure cette agonie de cet ancien vagabond qui semblait, après des siècles, le revanche de l'agonie inconnue et misérable de François Villon.

«Mais les fascistes, eux, ont vu mourir à Salamanque le seul et le plus grand des écrivains d'Espagne qui les ait rejoints - Miguel de Unamuno, destitué par eux, abandonné et solitaire.»

C'est à propos de culture que M. Malraux en vient à parler des rapports du gouvernement républicain avec l'Eglise catholique. 


\subsection{Les catholiques en Espagne}

«Parlons de l'Eglise catholique, dit-il. Pour l'Espagne, il serait plus juste de parler des Eglises, car les rapports entre le gouvernement et l'Eglise varient suivant les provinces. Les loyalistes fondent leur conception de l'Etat sur la liberté de conscience religieuse, sur la liberté des cultes. Tous ce qu'ils demandent, c'est la différenciation du temporel et du spirituel. Tout ce qu'ils demandent au prêtre, c'est de ne pas faire de politique.

«Il y a par exemple l'Eglise catholique basque. Elle s'est placée au-dessus de toute politique et elle est restée fidèle au gouvernement légitime, élu par le peuple. Elle considère que son unique devoir est le salut des âmes, et elle s'y cantonne en respectant l'autorité de l'Etat. Elle jouit en retour du respect le plus absolu du gouvernement de Madrid, et j'en donne entre autres preuves le fait que les évêques et les prêtres basques continuent de toucher régulièrement leur traitement - leur "salaire" - du gouvernement.

«D'autre part, il y a l’Eglise catalane. Celle-là, il est indéniable qu'elle rencontre en Catalogne une haine profonde. Ce sentiment, je n'ai pas à le juger. Mais, il y a quelques siècles, l'Espagne était un des premiers pays d'Europe. Si elle est devenue cette terre de misérables et l'illettrés, est-ce la faut des paysans espagnols ou de ceux qui les dirigeaient ? Tout ce que l'Eglise catalane reproche aujourd'hui, moralement, au peuple, qui donc en est responsable? Qui donc avait la charge de ces âmes depuis cinq cents ans? Et si elles ont appris la haine, c'est peut-être qu'on a oublié de leur enseigner l'amour.

«On ne doit pas oublier, continue M. Malraux, que le pape n'a jamais excommunié l'Eglise catholique basque, bien qu'elle soit restée fidèle à Madrid. Les évêques et les prêtres basques, tout aussi bien que les fidèles, continuent d'obéir à l'autorité spirituelle de Rome. Le pape n'a pas davantage condamné les catholiques laïques combattant du côté loyaliste, et tant que Pie XI ne se sera pas prononcé ex-cathedra, l'attitude du clergé et des fidèles en Espagne restera une affaire entièrement personnelle et d'ordre spirituel.»

Comme exemple typique, M. Malraux cite le cas de José Bergamín, le plus grand des écrivains catholiques d'Espagne, catholique pratiquant, et qui est président de la Société des Ecrivains et Intellectuels républicains.

\subsection{Mussolini, fauteur de guerre civile en Espagne}

«La guerre civile est essentiellement une intervention de Mussolini en Espagne, et cela en prévision d'une guerre contre l'Angleterre.

«Depuis un an deux découvertes ont laissé stupéfaits les experts militaires : la faiblesse marquée de l'aviation allemande de bombardement ; la faiblesse inattendue de la flotte anglaise.

«Les Allemands travaillent nuit et jour à reconstruire leur aviation, qui ne sera pas prête avant sept mois. Quant à la flotte anglaise, lorsque, pendant la guerre d'Ethiopie, 
la Home Fleet passa dans la Méditerranée, les experts, atterrés, découvrirent qu'elle était restée stationnaire alors que la flotte italienne était devenue une flotte moderne. L'Angleterre reconstruit sa flotte, qui sera prête avant un an.

«Mais si Mussolini tient le détroit de Gibraltar, l'Angleterre ne peut attaquer l'Italie par mer. L'Angleterre doit alors trouver un allié en Europe, ce qui implique la guerre européenne. Or, les chances de Mussolini sont infiniment plus grandes dans une guerre européenne que dans une guerre de l'Italie seule contre l'Angleterre après la reconstruction de la flotte anglaise. Il faut donc, en prévision de ce conflit européen, que l'Italie commence par tenir Gibraltar, et c'est la première étape de la politique mussolinienne.

«C'est pourquoi on a pu produire à la Société des Nations les instructions données à Franco par le consul italien de Tanger cinq jours avant la révolte fasciste - remarquez bien : je dis cinq jours avant, non pas après. Ce n'est pas le fascisme qui a volé su secours de Franco dans cette affaire, mais Franco qui a aidé le fascisme à atteindre ses fins. Et le fascisme espagnol paie au fascisme italien, avec le sang du peuple espagnol, la garantie de l'empire d'Ethiopie.»

\subsection{Les anecdotes épiques}

«Le jour du soulèvement de Franco à Barcelone, dit-il notamment, les insurgés avait placé sur la place de Catalogne les canons dont ils disposaient. Les ouvriers n'avaient pas de canons. Les ouvriers n'ont jamais de canons. Mais les chauffeurs syndicalisés du taxi prirent dans les garages des rues voisines toutes les Cadillac, placèrent dans chacune six camarades avec des révolvers, et ils lancèrent les autos de toute leur vitesse contre les canons. Les canons furent pris, les chauffeurs sont encore sur la place de Catalogne - mes les fascistes n'y sont plus.

«Un jour, dit-il, passant avec un camarade sur la route de Talavera, j'aperçus des quantité de bombes lancées par les avions fascistes, et qui n'avaient pas éclaté. Des camarades enlevaient les détonateurs, mais ils avaient découvert que ces bombes étaient inoffensives. C'étaient des bombes expédiées du Portugal aux troupes de Franco. A l'intérieur des bombes, les camarades portugais travaillant aux munitions, au lieu de mettre des explosifs, avaient mis ce message: “Cette bombe-ci n'éclatera pas."»

\subsection{Quand les camarades meurent}

Le 27 décembre, raconte ensuite M. Malraux dans une émouvante envolée, un des avions de mon escadrille fut abattu dans la région de Teruel, dans nos lignes. Il était tombé de très haut, à 2,000 mètres, dans une neige semblable à celle qui, semble-t-il, a dî abondamment recouvrir Montréal encore tout récemment. 
«Dans cette région, il y a très peu de villages. C'est seulement après quelques heures que les paysans arrivèrent et commencèrent à construire les brancards pour les blessés et le cercueil pour le mort.

«Quand tout fut prêt, la descente commença. Pas de route, des sentiers de mulet. Les vieilles paysannes qui, dans cette région, ont presque toutes un fils dans les milices, avaient décidé d'accompagner les blessés. Mais ce n'étaient pas seulement celles-là, c'était tout le village qui suivait derrière, en file dans le sentier étroit. A chacun des villages où nous passions, la population attendait ; et à chacun, quand elle avait vu arriver les blessés, elle suivait.

«Quand nous arrivâmes dans la vallée, au premier gros bourg, tous les paysans debout attendaient sur le rempart bas des villages espagnols. Les premiers blessés les blessés aux jambes - arrivèrent. Les paysans regardaient et ne disaient rien. Mais quand arrivèrent les blessés à la face, avec leurs pansements plats à la place du nez et leurs cuirs d'aviateurs encore recouverts de sang coagulé, les femmes et les enfants commencèrent à pleurer.

«Je relevais la tête: la file des paysans s'étageait maintenant du haut en bas de la montagne. Et c'est la plus grande image de la fraternité que j'aie rencontrée de ma vie, ces villages abandonnés, ce peuple entier qui suivait des hommes blessés pour lui, et qu'il n'avait jamais vus, descendant comme une processions antique, avec ces sanglots qui montaient dans le grand silence des gorges - avec le bruit d'une rivière souterraine.

«Les aviateurs fascistes blessés le même jour recevaient une escorte militaire. Et je pensais que précédemment, chacun des hommes couchés sur ces civières avait accepté de risquer sa vie pour que ce ne fut pas une escorte militaire, mais la virile fraternité du peuple même qui puisse accompagner désormais ceux qui combattent pour la peuple et pour un idéal de dignité humaine.»

\section{DISCOURS DE MONTRÉAL, 14 OCTOBRE 1963}

«Cette France qui vous parle aujourd'hui...»"

Voici le texte intégral de l'allocution prononcée lundi, le 14 octobre, par M. André Malraux, à l'issue du déjeuner offert par la ville de Montréal au ministre chargé des Affaires culturelles, à l'île Ste-Hélène. Rappelons au lecteur que M. Malraux ne lit jamais de texte. Brillante improvisation, son discours a été sténographié par les services de l'ambassade de France. (NDLR du Devoir)

Tout le monde sait que le destin du monde est en train de se jouer entre deux forces immenses: celle des Etats-Unis et celle de l'Union Soviétique. Ce qu'on voit moins bien, c'est que le domaine des cultures, au sens cette fois le plus élevé de tous, au sens de la conscience humaine, est en train de se jouer non pas entre deux forces, mais entre

\footnotetext{
${ }^{5}$ Nous donnons ici le texte paru dans Le Devoir le 18 octobre 1963, mais en nous permettant d'en établir autrement la série des paragraphes et en normalisant certains détails (usage de la majuscule, etc.). (Ndlr.)
} 
un certain nombre de forces. Je mets à part l'Asie qu'il faudrait analyser de plus près, et nous ne sommes pas là pour ça, et je retiens ce qui nous intéresse tous.

Il y a, d'une part, la culture américaine. Il y a, d'autre part la culture communiste. Et il y a, enfin, quelque chose qui ne veut être ni américain ni communiste. Non pas parce qu'il y a des gens dans des coins qui voudraient s'arranger doucement sur des choses sans importance, mais parce que ces gens qui ont fait la civilisation occidentale sentent qu'ils ne peuvent être ni dans cette droite ni dans cette gauche, sans y perdre ce qui fut leur âme.

Or, prenez bien garde, la première catégorie, c'est, en gros, moins le monde anglosaxon que l'ancien monde protestant. La seconde catégorie, si stupéfiant que ce soit - car Dieu sait que Lénine ne revendiquait pas essentiellement saint Jean Chrysostome -, c'est le monde de Byzance, c'est la vieille orthodoxie. Il n'y a de force communiste que là où la Russie est l'héritière de Byzance. Et le génie russe a été, comme me le disait Staline, de conjuguer Sparte et Byzance. Mais lorsque ça n'est pas Byzance, par exemple en Pologne, ça ne va pas très bien, et lorsque ça n'est pas Byzance du tout, par exemple en Chine, ça va extrêmement mal. Là où le communisme est réellement une puissance incarnée, enracinée, c'est dans le vieux domaine orthodoxe et byzantin. Et chose étrange, car je ne suis pas ici un défenseur de tel ou tel point de vue religieux, mais un historien, tout ce qui est en train de tenter de se réunir depuis cette ville jusqu'à ce qui se passe à Brasilia, à Saint-Paul et dans l'Europe tout entière, dans l'Europe occidentale, dans cette Europe occidentale à laquelle l'Angleterre a tant de mal à s'intégrer, eh bien! ce monde, c'est le vieil héritage catholique.

Quelle conséquence en tirerons-nous? Je laisse chacun choisir, et peut-être n'y a-t-il pas de conséquence à en tirer, car il serait tout à fait déraisonnable de croire que la puissance communiste tient à un lien avec Byzance. Mais ce que je veux retenir pour nous tous, c'est que le destin du monde, qui est notre destin, est en train de se jouer dans de vastes aires qui ne sont pas seulement culturelles et que tout se passe comme si le monde qui veut retrouver sa conscience entre les Etats-Unis et la Russie, c'était très simplement l'héritage de la Rome impériale et l'héritage de la Rome pontificale. Est-ce que nous retrouverons ensemble la Rome Impériale ? C'est simplement ridicule. Il ne s'agit pas de ça. Il s'agit simplement de comprendre où le domaine véritablement séculaire commence et probablement les motifs irrationnels qui font que nous sommes engagés sur une voie qui, à première vue, était assez obscure. Autrement dit, pourquoi le Canada, le Brésil, la France, la Grèce et l'Italie, et même l'Allemagne fédérale et catholique, à l'heure actuelle, sont-ils des pays qui ont partie liée, alors que sous les apparences de Marché Commun - on trouve toujours un Marché Commun quand l'âme en a besoin -, d'autres choses ne s'arrangent jamais ? Donc disons-nous qu'aujourd'hui en face, vous l'avez très bien dit, Monsieur le Maire, d'un problème américain que nous ne devrons pas du tout concevoir comme un problème ennemi - car l'Amérique 
est un des piliers du monde libre -, la conclusion de tout cela d'ici 20 ans, 30 ans ou 50 ans sera peut-être enfin l'organisation du monde libre. Mais enfin en face de cette réalité américaine, le Canada veut être autre chose. Pourquoi? Parce qu'il est autre chose, et que cette autre chose, c'est la même que j'ai constatée quand j'étais à Brasilia, et je revois de président de la République brésilienne qui venait de faire l'acte le plus saisissant qu'on ait fait depuis 25 ans, créer une ville admirable dans le désert, qui me disait : «Je veux faire cette ville parce que je veux que nous soyons nous-mêmes», et chose étonnante, nous c'étaient eux (et nous, les Français et vous ${ }^{6}$ ), "parce que nous voulons être nous-mêmes», et j'ajoute (c'est lui qui parlait): «Parce que nos femmes ne sont pas des Américaines»!

Alors le problème réel, il est, non pas un Tiers Monde, mais bien nous: nous qui sommes ce à partir de quoi s'est fondé ce qu'on appelle la civilisation occidentale. Ce que sous sommes est en train de prendre forme, comme le désordre russe et américain a cessé il y a 30 ou 40 ans. Nous avions 1500 ans d'avance; nous avons probablement 40 ans de retard. Cela peut se rattraper. En fait, les Etats-Unis et la Russie sont entrés ensemble dans l'Histoire. Au XVIII siècle, il n'y avait pas d'Amérique ; il n'y avait pas de Russie dans l'Histoire universelle. En deux siècles, l'un et l'autre sont devenus ce que nous savons. Aujourd'hui commence dans l'Histoire du monde la volonté d'un certain nombre de pays de la vieille Europe, peut-être parce qu'ils sont d'origine catholique, ou pour d'autres raisons, d'être ce qu'ils sont et de donner forme à leur âme et à leur rêve. C'est là dessus que nous nous unissons.

Vous avez un certain nombre de gratte-ciel et cette ville est extrêmement saisissante. Bien entendu, nous n'allons pas décider qu'il serait raisonnable de supprimer les gratte-ciel. Les gratte-ciel ne sont pas nés d'une psychologie des Etats-Unis. Ils sont nés de l'union du béton et de l'ascenseur et du prix des terrains dans certains endroits. Ce qui est important, ce n'est pas du tout de supprimer les gratte-ciel, c'est de faire des gratte-ciel qui vous appartiennent. L'architecture moderne des Etats-Unis est incontestablement la première du monde. A deux conditions: premièrement, à la seule exception du Rockefeller Center, elle n'a jamais fait que des constructions en forme de maisons. Elle n'a jamais fait un édifice, un ensemble, un palais, ce que nous admirons sous le nom de Louvre, ou d'un bout à l'autre de l'Italie, ou encore ce que nous admirons sur l'Acropole. La civilisation moderne n'a su créer ni un temple ni un tombeau, mais les constructeurs de Broadway non plus. En conséquence, ce qui est important serait que vous disiez: «Nous, les Canadiens, nous allons faire un ensemble. Puisque à Montréal, nous avons du terrain, de l'espace - au lieu de faire les deux ou trois édifices que vous avez faits et qui sont assez dignes de respect -, nous allons en faire dix. Mais nous allons en faire dix ensemble et, comme les Brésiliens ont fait Basilia, nous allons

\footnotetext{
${ }^{6}$ Passage particulièrement obscur que seule l'audition d'un enregistrement du discours pourrait atténuer. Et si la sténographe avait mal compris ce qui pourrait être: «et vous les Français et nous»? (Ndlr.)
} 
faire un Montréal dans lequel les Canadiens pourront arriver en disant: "Gratte-ciel ou pas gratte-ciel, c'est nous qui l'avons fait et personne en Amérique ne l'avait tenté".»

L'âme de ce pays, je la vois naître sur un autre plan, que certains d'entre vous voient comme moi. Pour d'autres. Ils penseront peut-être que le rêve... - Monsieur le Ministre Lapalme me passait hier une revue éditée ici dans laquelle les poètes parlaient de Montréal avec amour. Mesdames et Messieurs, comprenez bien que, à partir du moment où l'amour existe dans la littérature pour quelque chose, il y a une véritable naissance. Qu'est-ce que Rome illustre dans l'Histoire? C'est le passage d'un certain nombre de poètes. Qu'est-ce que Paris? Ce sont des quais sur lesquels se promenaient Victor Hugo et Baudelaire. Et Notre-Dame de Paris, bien sûr, c'est Notre-Dame et tant de sacres de ce lieu, c'est aussi Notre-Dame de Paris de Victor Hugo. Vos poètes sont en train de commencer à aimer votre ville. A partir du moment où l'amour naît quelque part, c'est l'âme collective qui commence.

Maintenant, Monsieur le Maire, vous avez bien voulu dire de mon pays des choses dont, pour la seconde fois, je vous remercie. Je vais essayer d'en parler à mon tour de la façon la plus courte possible. Il y a des pays qui sont grands dans l'Histoire quand ils se replient sur eux-mêmes. Ils sont alors exemplaires, et c'est avant tout le cas de la Grande-Bretagne. Il n'est pas dans mon esprit d'ignorer ce que fut la bataille de Londres : le moment où les aviateurs anglais remontaient avec un œil crevé ou un bras de moins sur les derniers avions anglais pour qu'à la dernière minute l'aviation allemande fût battue, est un des moments qui appartiennent au sommet de l'Histoire du monde. Mais l'Angleterre n'est grande que lorsqu'elle est elle-même. La France n'est réellement grande que lorsqu'elle est la France pour tous les hommes. Sur tous les chemins de la Chrétienté, sur tous les chemins de l'Orient des croisades, il y a des tombeaux de chevaliers français. Sur toutes les routes de la Liberté, il y a des cadavres de soldats français. C'est ce que la France signifie ou ce qu'elle veut signifier quand elle est la France. Je résume en citant la dernière phrase des Mémoires du général de Gaulle": «Avec nos forces et nos faiblesses, tels que nous sommes, Mère, pour te

\footnotetext{
${ }^{7}$ Malraux cite de mémoire et approximativement, comme il en a l'habitude, le dernier paragraphe de L'Appel, tome I ${ }^{\text {er }}$ des Mémoires de guerre. «Trêve de doutes! Penché sur le gouffre où la patrie a roulé, je suis son fils, qui l'appelle, lui tient la lumière, lui montre la voie du salut. Beaucoup, déjà, m'ont rejoint. D'autres viendront, j'en suis sûr ! Maintenant, j'entends la France me répondre. Au fond de l'abîme, elle se relève, elle marche, elle gravit la pente. Ah! mère, tels que nous sommes, nous voici pour vous servir.» De Gaulle, Mémoires, éd. de M.-F. Guyard, Paris, Gallimard, 2000, (coll. «Bibliothèque de la Pléiade»), p. 262.

Ce ton inspiré de Péguy se trouvait déjà dans quelques discours de De Gaulle. Celui du 27 juin 1943 («Discours prononcé à Tunis») se termine par ces phrases : «A la France, à notre Dame la France, nous n'avons à dire qu'une seule chose, c'est que rien ne nous importe ni ne nous occupe, excepté de la servir. Notre devoir envers elle est aussi simple et élémentaire que le devoir des fils à l'égard d'une mère opprimée. Nous avons à la délivrer, à battre l'ennemi et à châtier les traîtres qui l'ont jetée dans l'épreuve, à lui conserver ses amis, à arracher le bâillon de sa bouche et les chaînes de ses membres pour qu' elle puisse faire entendre sa voix et reprendre sa marche au destin.» De Gaulle, Discours et messages, t. I ${ }^{\text {er: }}$ Pendant la guerre, 1940-1946, Paris, Plon, 1970 [1946], p. 309. Le discours du 18 juin 1942 se terminait
} 
servir». Canadiens, c'est cette France qui vous parle aujourd'hui telle qu'elle fut, telle qu'elle est, tels que nous sommes, avec nos mains périssables. «A ton service, Mère, pour servir le monde.» Puissiez-vous, Canadiens, le servir avec nous! ${ }^{8}$

\section{BIBLIOGRAPHIE}

«André Malraux à Montréal» Revue André Malraux review, vol. 19, nos 1-2, printempsautomne 1987 - vol. 20, n 1, printemps 1988 : «De 1'“escadrille España” à L'Espoir. Essais sur Malraux et la guerre civile espagnole», 165-171. (Reprise d'un compte rendu paru dans Le Devoir le 5 avril 1937.)

"André Malraux à New York, Los Angeles, San Francisco» Revue André Malraux review, vol. 19, nos 1-2, printemps-automne 1987 - vol. 20, n²1, printemps 1988 : «De l'“escadrille España” à L'Espoir. Essais sur Malraux et la guerre civile espagnole», 159-164. (Reprise de comptes rendus parus en 1937 dans La France d'aujourd'hui.)

DESY, C. (2011) : «Malraux à Montréal : tensions et démesure autour d'une visite», Présence d'André Malraux sur la Toile, art. 115. URL : <http:/ / www.malraux.org/index. php/articles/1507-art115.html>.

DE GAULLE, Ch. (1970) : Discours et messages, t. Ier, Pendant la guerre, 1940-1946, Paris, Plon.

DE GAUlLE, Ch. (2000) : Mémoires, éd. de M.-F. Guyard, Paris, Gallimard. (coll. «Bibliothèque de la Pléiade»).

MALRAUX, A. (1937): "Devant les publics les plus divers d'Amérique André Malraux a parlé de l'Espagne», entretien accordé à Edith Thomas, Ce Soir [Paris], 1re année, $\mathrm{n}^{\circ}$ 52, 22 avril 1937, 3.

" "La France n'a de remords qu'à l'égard du Canada français"», compte rendu, par Yves Margraff, d'une conférence de presse accordée le 7 octobre 1963, à l'aéroport de Montréal. Le Devoir [Montréal], 9 octobre 1963.

"L'Allocution d'André Malraux», discours prononcé le 10 octobre 1963 à l'hôtel de ville de Montréal. Le Devoir [Montréal], 12 octobre 1963. Des extraits avaient paru dans le compte rendu de Jean-Marc Léger et de Jean-Marc Laliberté, «Malraux: "Canadiens français, nous ferons ensemble la civilisation de demain..." ", Le Devoir [Montréal], 11 octobre 1963.

"L'exposition française de Montréal est ouverte. Malraux : fairelacivilisationdel'Atlantique», compte rendu, par Marc-Henri Côté, de l'allocution prononcée par Malraux à l'occasion de l'inauguration de l'Exposition industrielle et économique française de Montréal, au Palais du commerce, le 11 octobre 1963. Le Devoir [Montréal], 12 octobre 1963.

d'ailleurs par cette citation de Péguy: «Mère, voici vos fils, qui se sont tant battus.» De Gaulle, op. cit. p. 204. (Ndlr.)

8 Malraux, «Cette France qui vous parle aujourd'hui...», Le Devoir (Montréal), 18 octobre 1963. 
«Malraux : la France peut vous apporter la confiance en vous», compte rendu de l'allocution prononcée au dîner offert par le gouvernement du Québec au château Frontenac, le 11 octobre 1963. Le Devoir [Montréal], 14 octobre 1963.

«La France vous tend la main en face de l'avenir...», allocution prononcée le 11 octobre 1963 à l'issue du déjeuner offert par l'ambassade de France et la chambre de commerce française, à l'hôtel Ritz-Carlton, à Montréal. Le Devoir [Montréal], 15 octobre 1963. "Cette France qui vous parle aujourd'hui...», allocution prononcée le 14 octobre à l'issue du déjeuner offert par la ville de Montréal à l'île Ste-Hélène. Le Devoir [Montréal], 18 octobre 1963.

«Discours prononcé à l'université de Montréal, le 15 octobre 1963», La Presse [Montréal], 16 octobre 1963.

CEuvres complètes, t. II, éd. de M.-F. Guyard, Paris, Gallimard, 1996, (coll. «Bibliothèque de la Pléiade»).

CEuvres complètes, t. III, éd. de M.-F. Guyard, Paris, Gallimard, 1996, (coll. «Bibliothèque de la Pléiade»).

LANGLOIS, W G. (1979) : «Prélude to L'Espoir : Malraux in the United States (Spring 1937)», Wascana Review, n 14, printemps 1979 : «Malraux», 1-21.

"Malraux au service de la République espagnole : un mois à New York (février-mars 1937)», in LANGLOIS, Walter G. [édit.], André Malraux, t. 5 : Malraux et l'histoire, Paris, Lettres modernes Minard, 1982, (coll. «La Revue des lettres modernes», nos 643647), 93-114.

THORNBERRY, R S. André Malraux et l'Espagne, Genève, Droz, 1977, (coll. «Histoire des idées et critique littéraire», $\left.\mathrm{n}^{\circ} 166\right)$.

«André Malraux. Republican Propagandist in Canada (1937)», Wascana Review, vol. 14, $\mathrm{n}^{\circ}$ 1, printemps 1979 : «Malraux», 22-36.

«L'antifascisme de Malraux (1932-1938) : réflexions sur une "passion négative"», in CHAMI, Anissa B. [édit.], Quêtes d'un idéal humain et de valeurs transcendantes, actes du colloque international organisé par le centre de Recherches Méditerranéennes, Casablanca, 25-27 février 2004. Casablanca, A. Retnani édition - La croisée des chemins, 2006, 53-65.

SEYLAZ, J-L. (1974): "Malraux : "La Descente de la montagne", in La Quintefeuille. Cinq études sur Balzac, Nerval, Flaubert, Malraux, Robbe-Grillet, Lausanne, L'Aire, 91-121. 